\title{
Controlled placement of highly aligned carbon nanotubes for the manufacture of arrays of nanoscale torsional actuators
}

\author{
T D Yuzvinsky ${ }^{1,2,3}$, A M Fennimore ${ }^{1,2}$, A Kis ${ }^{1,2}$ and A Zettl ${ }^{1,2,3,4}$ \\ ${ }^{1}$ Department of Physics, University of California at Berkeley, Berkeley, CA 94720, USA \\ ${ }^{2}$ Materials Sciences Division, Lawrence Berkeley National Laboratory, Berkeley, CA 94720, \\ USA \\ ${ }^{3}$ Center of Integrated Nanomechanical Systems, Berkeley, CA 94720, USA \\ E-mail: azettl@berkeley.edu
}

Received 15 September 2005

Published 14 December 2005

Online at stacks.iop.org/Nano/17/434

\begin{abstract}
We have fabricated ordered arrays of nanoscale torsional actuators consisting of metal mirrors bonded to precisely oriented multiwall carbon nanotubes. The fabrication is facilitated by a new nanotube positioning method which employs localized electron beam activation of polymer residue on a silicon oxide surface.
\end{abstract}

\section{Introduction}

Recent advances in nanoscale synthesis and fabrication techniques have opened the door to the manufacture of true nanoelectromechanical systems (NEMS). For example, multiwall carbon nanotubes (MWCNTs) have been utilized as key enabling elements for nanoscale electrostatically driven torsional [1] and rotational [2] actuators, orders of magnitude smaller than their microelectromechanical (MEMS) counterparts. Due to their small size, robust design and near-perfect atomic structure, such constructs hold great promise as building blocks for complex nanoelectromechanical systems. The utility of individual actuators can be significantly increased by their incorporation into arrays of devices. Such arrays could serve in a variety of applications, including adaptive optics, high frequency mechanical filters, mass sensors, and microfluidic gates and pumps.

A fundamental challenge in the development of NEMS arrays (and of nanotube- and nanowire-based devices in general) is the large-scale controlled placement of molecular sized building blocks on a substrate. Methods based on chemical vapour deposition (CVD) avoid this problem by, for example, growing nanotubes directly on the substrate where they ultimately will be located [3]. Unfortunately, such methods are unable to produce very high quality multi-walled carbon nanotubes as are often required for

4 Author to whom any correspondence should be addressed.
NEMS applications [2, 4, 5]. Furthermore, CVD is commonly a high temperature process, which severely limits compatibility with substrate materials or other system components. Hence there is much interest in low temperature techniques to aid in the selective placement and alignment of prefabricated nanostructures. There has been some progress in developing fluidic techniques for aligning nanowires [6] and nanotubes [7-9], and various functionalization schemes have been explored for placing nanotubes on particular areas of a substrate [10-12]. Unfortunately, most of these methods necessitate rather complex substrate topology or involved and limiting chemistry.

We have developed a combination of novel roomtemperature methods for both aligning and selectively depositing nanotubes onto a topologically benign surface. Using these methods, which can easily be integrated into semiconductor manufacturing processes, we have fabricated arrays of aligned torsional NEMS devices based on MWCNTs.

\section{Fabrication process}

Figure 1 outlines the fabrication process. High quality MWCNTs are grown using the arc-plasma method and are dispersed in solution. A silicon/silicon oxide substrate is coated with electron beam resist, which is then soaked in acetone to leave only a very thin polymer layer. The substrate is then selectively exposed to an electron beam in situ in an FEI Sirion XL30 scanning electron microscope, which activates 


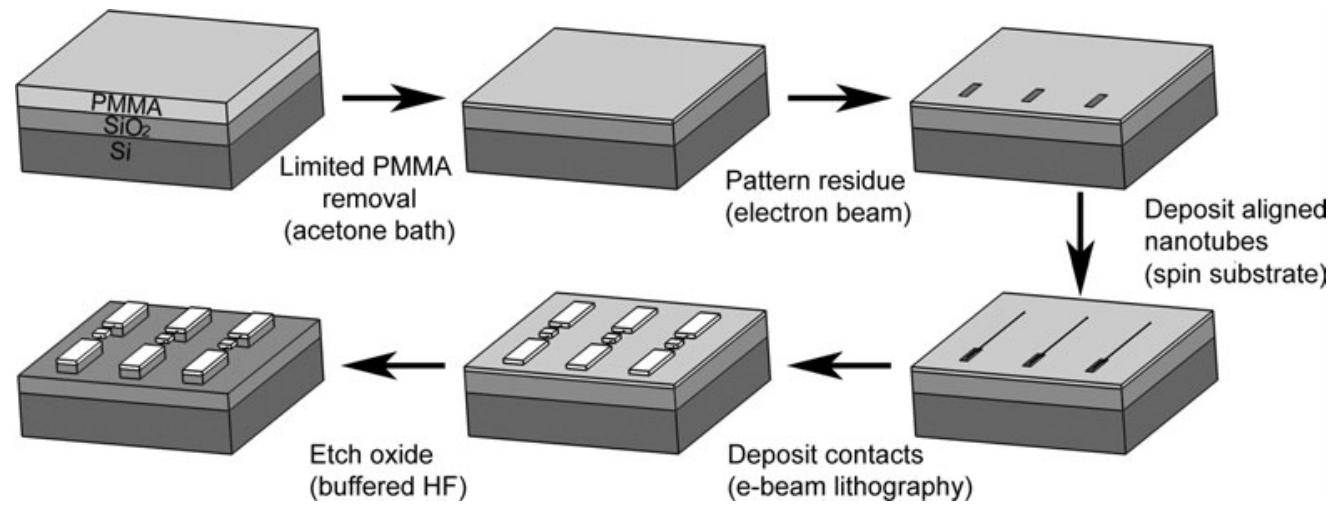

Figure 1. Fabrication process. A silicon/silicon oxide wafer is coated with electron beam resist (not to scale) which is then mostly removed in an acetone bath. The remaining residue is then patterned by exposure to an electron beam in situ in an SEM. MWCNTs are deposited out of solution onto the spinning substrate, causing them to align with fluid flow and adhere to the exposed pattern. Standard electron beam lithography is then used to deposit anchors and paddles. Finally, the silicon oxide is partially etched down to suspend the nanotube-paddle assemblies.

the adhesive properties of the polymer layer. The MWCNT solution is then deposited onto the spinning substrate, causing the MWCNTs to align with the flow and adhere in the targeted regions. The alignment and adhesion parameters are discussed in detail in the next section. Finally, standard electron beam lithography is used to pattern paddles and anchors on top of the MWCNTs, which are then suspended by etching down the supporting oxide layer with buffered hydrofluoric acid.

\section{Results and discussion}

Figure 2(a) shows one section of a prototype array of torsional actuators that we have produced. An individual actuator, as shown in figure 2(b), consists of five main elements: two anchors, a suspended MWCNT, a suspended rectangular metal mirror or paddle, and the conducting back gate buried beneath the surface. The suspended MWCNT serves both as the torsional element and the electrical connection to the gold paddle mounted at its centre. The two gold anchors hold the ends of the MWCNT in place. Asymmetric electrostatic fields applied between the gold paddle and the back gate create an attractive force which causes the paddle to undergo an angular displacement and the MWCNT to twist. If the outer MWCNT shell is compromised, free rotational motion of the actuator becomes possible.

Actuators of the type shown in figure 2 have been previously individually fabricated and characterized $[1,2,13]$. Effective torsional spring constants range from $10^{-15}$ to $10^{-12} \mathrm{~N} \mathrm{~m}$, depending on the MWCNT geometry. Typical devices have moments of inertia $\sim 10^{-30} \mathrm{~kg} \mathrm{~m}$, yielding resonance frequencies in the tens of megahertz range (with smaller paddle sizes and shorter exposed MWCNT lengths the resonance frequencies can be extended to above $1 \mathrm{GHz}$ ). The key advance of the present work is the ability to produce such devices in an array configuration.

We describe in detail the key enabling step in the array fabrication process, namely MWCNT targeted deposition and alignment. Our alignment method exploits the surface velocity obtained by a fluid as it flows off of a spinning substrate, and is distinct from previous alignment attempts involving either dielectrophoresis [14] or deposition from a solution driven across a surface by gas [7, 8] or microfluidic flow [6]. We use arc-grown MWCNTs with typical diameters of 10-25 nm as measured by transmission electron microscopy (TEM). The MWCNTs are suspended in orthodichlorobenzene (ODCB) at a concentration of $100 \mathrm{mg} \mathrm{l}^{-1}$ by ultrasonication in a VWR model 75D Aquasonic bath for $60 \mathrm{~s}$ at level 3. TEM observation shows that this level of ultrasonication is enough to separate most MWCNT bundles into individual MWCNTs. The suspension is then pipetted drop by drop onto the centre of a silicon substrate mounted on a spin coater rotating at $3000 \mathrm{rpm}$. The suspension flows radially across and off the substrate. Once the surface is dry, the next drop is deposited. We find that if the next drop is deposited while the substrate is still wet, the deposition is less dense and contains a larger percentage of unaligned nanotubes.

Figure 3 shows the results of a typical deposition/alignment run. MWCNTs are observed to align with the fluid flow direction (from lower right to upper left in the figure), with longer MWCNTs generally more aligned than shorter segments. From SEM images we observe that typically $90 \%$ of MWCNTs over $1 \mu \mathrm{m}$ in length lie within $\pm 1^{\circ}$ and $95 \%$ lie within $\pm 5^{\circ}$ of the direction of fluid flow, a significant improvement over previously reported results [7, 8].

Although the MWCNTs of figure 3 are aligned, they are still randomly positioned. For many multi-component engineered NEMS devices, including arrays, pre-determined positional order is necessary. The deposition process can be further refined by locally treating the substrate to place MWCNTs in target locations. Previous targeting attempts via surface functionalization have been made using self-assembled monolayers (SAMs) with polar functional groups [10-12]. The success of these techniques is dependent upon the quality of the SAM, however, with degraded performance occurring when more (or less) than a monolayer is deposited [15]. Ideally, localized nanotube deposition should involve a surface layer which is simple to deposit and insensitive to variations in thickness.

Our location targeting method exploits a surface layer that is already present in standard lithographic work and does 


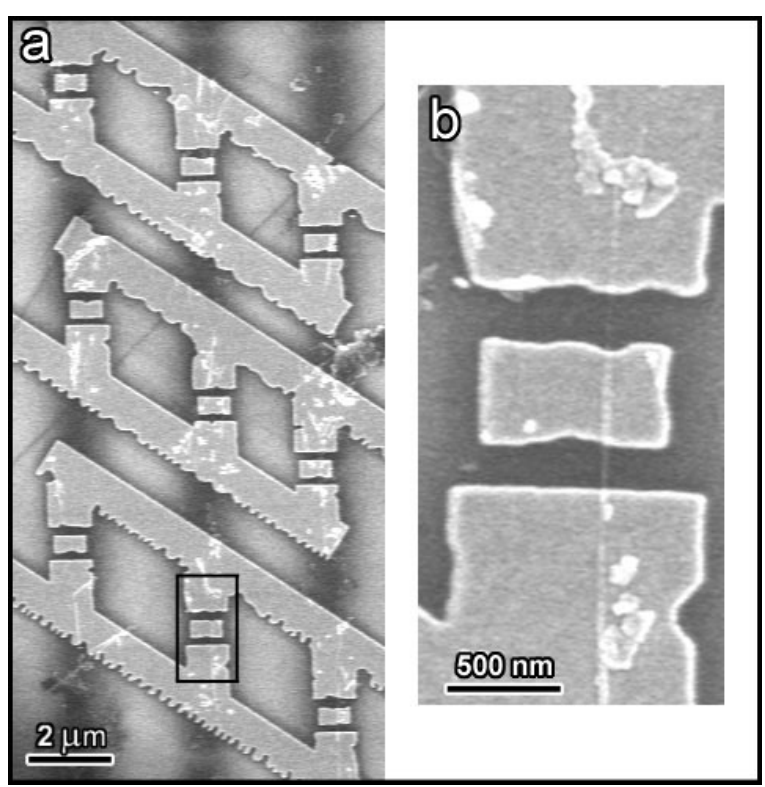

Figure 2. (a) An array of nanotube devices fabricated on MWCNTs placed by fluidic alignment and beam-activated adhesion shown prior to the HF etch. (b) Increased magnification image of the nanotube device marked with a black box in (a). The two anchors occupy the top and bottom thirds of the image, with the MWCNT support shaft running vertically between them. The paddle is mounted on the MWCNT in the centre of the image and can be driven by voltages applied to the conducting back gate (not visible).

not require a single monolayer. The layer consists of the residual polymer left behind when resists used in electron beam lithography are removed by an acetone wash. In this work, the resist consisted of a copolymer of $8.5 \%$ methacrylic acid (MAA) in methyl methacrylate (MMA) formulated in ethyl lactate at $6 \%$ (available from MicroChem Corp.), and was spincoated at $3000 \mathrm{rpm}$ for $30 \mathrm{~s}$ onto a silicon substrate with $1 \mu \mathrm{m}$ of thermally grown oxide. It was then baked on a hot plate at $185^{\circ} \mathrm{C}$ for $3 \mathrm{~min}$ and stripped in acetone for $5 \mathrm{~min}$. When left untreated, the residual coating actually inhibits nanotube deposition out of ODCB, as evidenced by lower deposition rates than those seen on pristine silicon substrates.

Targeted adhesion of nanotubes by this layer is activated by exposure to the low energy electron beam of a scanning electron microscope (SEM). Figure 4(a) shows the edge of a patterned area onto which nanotubes have been randomly deposited with no alignment. The preferential deposition of nanotubes on the selected area (lower half of the figure) is easily apparent. By controlling the raster of the electron beam we are able to create any desired adhesion pattern on the substrate, with the untreated residual coating inhibiting deposition elsewhere. By combining targeted adhesion and fluidic alignment, deposition of nanotubes in an ordered fashion becomes feasible. Figure 4(b) shows single-walled carbon nanotubes (SWCNTs) which have been deposited from a solution flowing parallel to patterned lines of activated substrate, while figure 4(c) is an example of MWCNTs deposited from a solution flowing at $\sim 70^{\circ}$ to patterned lines of activated substrate; here we observe that the trailing edge of the nanotubes adheres to the targeted region. If the activated regions are dots rather than lines, then a targeted array is

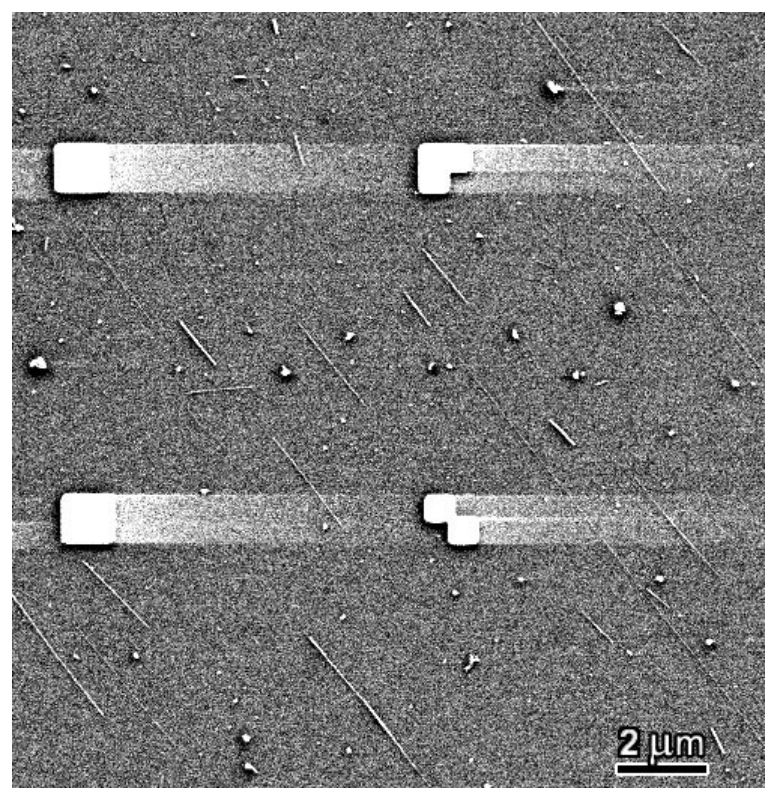

Figure 3. Fluidic alignment of MWCNTs by spinning the substrate. A suspension of nanotubes was pipetted onto this sample while it was spinning at $5000 \mathrm{rpm}$, with the centre of rotation $\sim 6 \mathrm{~mm}$ below and to the right of the area shown. The substrate was in its pristine state before deposition; alignment marks were patterned and deposited afterwards.
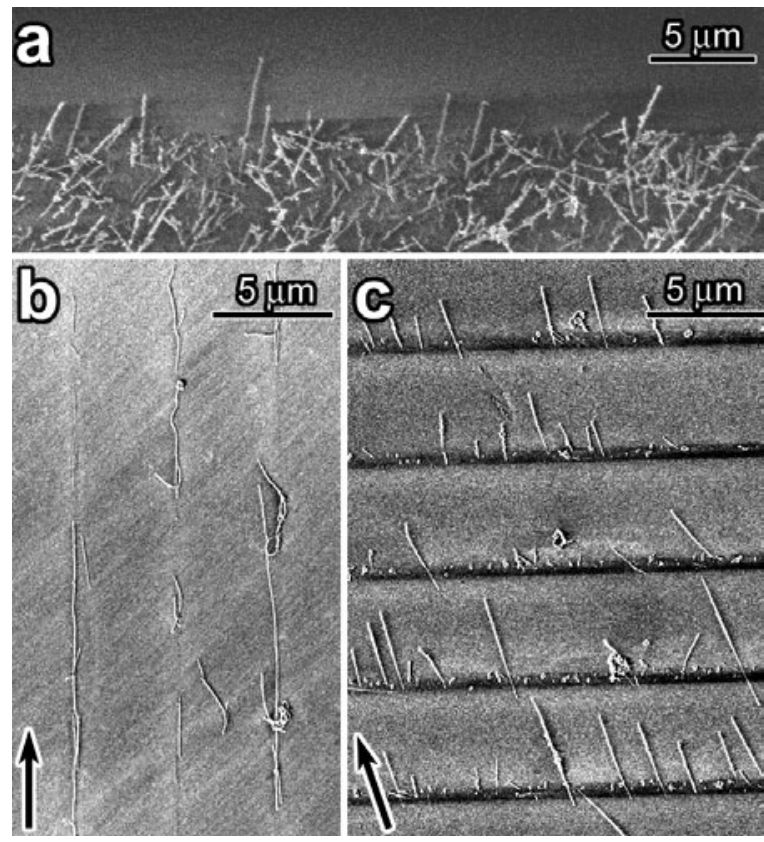

Figure 4. Controlled deposition of nanotubes. (a) The edge of an unaligned mat of MWCNTs deposited on an area activated by the electron beam. The edge of the beam-activated area runs horizontally through the centre of the image. (b) SWCNTs placed by fluidic alignment onto beam activated lines which were oriented parallel to the direction of fluid flow. (c) MWCNTs placed by fluidic alignment at $\sim 70^{\circ}$ to beam-activated lines. The arrows indicate the direction of flow.

possible, as is the case for the two-dimensional MWCNT array underlying the devices of figure 2. By changing the size and 
spacing of the dots we are able to control the average number of MWCNTs adhering to each dot. If non-purified nanotubes are used in the alignment and targeted deposition process, carbon onions and other by-products of the arc discharge process adhere to the beam activated areas as well.

To determine the source of selective deposition following electron beam activation, we have tested other surface coatings. Coatings of poly(methyl methacrylate) (PMMA) in anisole (2\% PMMA) and in chlorobenzene (3.5\% PMMA) produce similar results. Substrates with no polymer present (pristine wafers, or those which had been plasma cleaned or soaked for $12 \mathrm{~h}$ in acetone) show no evidence of selective adhesion following beam exposure.

Polymer chains in PMMA experience several structural transitions when exposed to increasing doses of electron irradiation. At the low doses normally used in electron beam lithography, the polymer chains undergo scission, allowing for their selective removal by an appropriate developer. At higher doses, nearby chains become cross-linked. For an acceleration voltage of $15 \mathrm{keV}$, cross-linking prevails over scission at doses of $\sim 1500 \mu \mathrm{C} \mathrm{cm}^{-2}$ [16]. As the dosage is further increased, a highly cross-linked network is formed $\left(\sim 10000 \mu \mathrm{C} \mathrm{cm}^{-2}\right.$ at $15 \mathrm{keV}$ ) [16]. We find that the minimum dose for selective nanotube adhesion is $5000 \mu \mathrm{C} \mathrm{cm}^{-2}$, with an optimal dose of $\sim 50000 \mu \mathrm{C} \mathrm{cm}^{-2}$.

Our measured doses, however, are only effective at low acceleration voltages $(\sim 1 \mathrm{keV})$, and correspond to much higher doses at $15 \mathrm{kV}$. Due to their higher interaction crosssection, low energy electrons have a higher stopping power than high energy electrons (stopping power is the rate at which electrons transfer energy to the material they are travelling through and has been studied extensively for many materials [17]). For carbon, the stopping power of $1 \mathrm{keV}$ electrons is roughly seven times greater than that of $15 \mathrm{keV}$ electrons [18]. Our minimum dose of $5000 \mu \mathrm{C} \mathrm{cm}^{-2}$ at $1 \mathrm{keV}$ is therefore far in excess of the equivalent cross-linking threshold dose discussed above, which suggests modification of the PMMA beyond simple cross-linking. PMMA has also been shown to graphitize when subjected to large doses of ion beam irradiation [19]. The lack of adhesion to cross-linked networks and the large effective doses being delivered are highly suggestive that the graphitization threshold has been reached. To our knowledge this is the first time this effect has been demonstrated with electron beam irradiation.

Targeted deposition is selective to the solvent used in the nanotube suspension. The effect is not observed for a majority of common solvents and solutions (dichloroethane, isopropanol, acetone, ethyl lactate, and $1 \%$ sodium dodecyl sulfate in water). Of the solvents tested, only ODCB (and to a lesser extent methoxybenzene) resulted in effective targeted deposition. Ultrasonication of SWCNT in ODCB has been shown to create a sonopolymer which coats the surface of the nanotubes [20]. Although our method requires less ultrasonication than was reported to cause significant coating by the sonopolymer, inspection of similarly dispersed MWCNTs by TEM has shown that while the nanotubes are still mostly pristine there are isolated sections covered with a thin amorphous coating. We suggest that polymerized solvent adhering to the nanotubes increases their interaction with the beam activated substrate-in effect, the nanotubes must be activated along with the substrate.
Once arrays of MWCNTs or similar structures have been deposited, further processing and device fabrication can be relatively straightforward. In our torsional actuator demonstration array, the nanotubes are first placed in an array configuration on a degenerately doped silicon wafer with $1 \mu \mathrm{m}$ of thermally grown oxide on its top surface. The paddle and anchors are then patterned by electron beam lithography and deposited by electron beam evaporation of gold. To suspend the devices, approximately $500 \mathrm{~nm}$ of the silicon oxide is selectively removed with a buffered hydrofluoric acid etch. In the prototype array shown in figure 2 , each row of actuators is connected together to simplify the wiring arrangement and still allow for semi-independent actuation. The actuators could be made completely independent by using a more complicated wiring scheme, perhaps incorporating multiple conducting layers to avoid shorting the leads from one device to another. Multilayer processing would also allow for higher density packing of devices.

Torsional actuator arrays operating at radio frequencies might find use in optical switching or in adaptive optics applications. With each actuator serving as a high frequency mechanical filter, such an array could also be used for parallel signal processing in telecommunications. Furthermore, by tracking the frequency shift of each actuator, an array of individually functionalized actuators could be used as mass sensors for simultaneous environmental monitoring of a variety of substances.

\section{Conclusions}

In summary, we have fabricated arrays of torsional actuators using a novel nanotube positioning technique. The nanotubes are oriented by fluid flow across a spinning substrate and adhere preferentially to regions that have been activated by a focused electron beam. The adhesion is found to be due to extreme modification of a residual polymer layer already present in lithographic work. By varying the flow direction and electron beam exposure we are able to deposit nanotubes in a variety of patterns that can then be contacted by standard lithography.

Our ability to orient and preferentially place molecular structures paves the way for their integration into mass produced devices. Aside from the torsional actuator array already described, an immediate, simple application is the use of nanotubes as electrical interconnects between units of an integrated circuit (especially desirable due to their ability to carry extremely high electrical and thermal current densities). The unique physical properties of carbon nanotubes could be harnessed in other applications as well, such as high density arrays of field effect transistors, gas sensors or biosensors. Alternative geometrical configurations, including radially aligned nanotubes, crossed nanotubes etc, could be easily fabricated using the techniques described here.

\section{Acknowledgments}

This work was supported in part by the Director, Office of Energy Research, Office of Basic Energy Sciences, Materials Sciences Division of the US Department of Energy, under contract No DE-AC-03-76SF00098, and by the National Science Foundation under grant No EEC-0425914. 


\section{References}

[1] Papadakis S J, Hall A R, Williams P A, Vicci L, Falvo M R, Superfine R and Washburn S 2004 Phys. Rev. Lett. 93146101

[2] Fennimore A M, Yuzvinsky T D, Han W Q, Fuhrer M S, Cumings J and Zettl A 2003 Nature 424408

[3] Tseng Y C, Xuan P Q, Javey A, Malloy R, Wang Q, Bokor J and Dai H J 2004 Nano Lett. 4123

[4] Cumings J and Zettl A 2000 Science 289602

[5] Salvetat J-P, Bonard J-M, Thomson N H, Kulik A J, Forro L, Benoit W and Zuppiroli L 1999 Appl. Phys. A 69255

[6] Huang Y, Duan X F, Wei Q Q and Lieber C M 2001 Science 291630

[7] Xin H J and Woolley A T 2004 Nano Lett. 41481

[8] Lay M D, Novak J P and Snow E S 2004 Nano Lett. 4603

[9] Hedberg J, Dong L and Jiao J 2005 Appl. Phys. Lett. 86143111

[10] Burghard M, Duesberg G, Philipp G, Muster J and Roth S 1998 Adv. Mater. 10584

[11] Liu J, Casavant M J, Cox M, Walters D A, Boul P, Lu W, Rimberg A J, Smith K A, Colbert D T and Smalley R E 1999 Chem. Phys. Lett. 303125
[12] Rao S G, Huang L, Setyawan W and Hong S H 2003 Nature $\mathbf{4 2 5} 36$

[13] Williams P A, Papadakis S J, Patel A M, Falvo M R, Washburn S and Superfine R 2002 Phys. Rev. Lett. 89255502

[14] Yamamoto K, Akita S and Nakayama Y 1998 J. Phys. D: Appl. Phys. 31 L34

[15] Valentin E, Auvray S, Goethals J, Lewenstein J, Capes L, Filoramo A, Ribayrol A, Tsui R, Bourgoin J P and Patillon J N 2002 Microelectron. Eng. 61/62 491

[16] Koval Y 2004 J. Vac. Sci. Technol. B 22843

[17] See for example NIST stopping power and range tables available at http://physics.nist.gov/PhysRefData/Star/Text/ contents.html

[18] Joy D C, Suichu L, Gauvin R, Hovington P and Evans N 1996 Scanning Microsc. 10653

[19] Davenas J, Thevenard P, Boiteux G, Fallavier M and Lu X L 1990 Nucl. Instrum. Methods Phys. Res. B 46317

[20] Niyogi S, Hamon M A, Perea D E, Kang C B, Zhao B, Pal S K, Wyant A E, Itkis M E and Haddon R C 2003 J. Phys. Chem. B 1078799 